\section{4 \\ DIAGNOSTIC ACCURACY OF EXERCISE STRESS TESTING IN INDIVIDUALS WITHOUT KNOWN CORONARY ARTERY DISEASE: A SYSTEMATIC REVIEW AND META-ANALYSIS}

doi:10.1136/heartjnl-2011-300198.64

${ }^{1} \mathrm{~A}$ Banerjee, ${ }^{2} \mathrm{D}$ Newman, ${ }^{2} \mathrm{~A}$ Van den Bruel, ${ }^{2} \mathrm{C}$ Heneghan. ${ }^{1}$ West Midlands Deanery, Birmingham, UK; ${ }^{2}$ Department of Primary Care, University of Oxford, Oxford, UK

Background Exercise stress testing offers a non-invasive, less expensive way of risk stratification prior to coronary angiography, and a negative stress test may actually avoid angiography. However, previous meta-analyses have not included all exercise test modalities, or patients without known coronary artery disease.

Objectives To systematically review the literature to determine the diagnostic accuracy of exercise stress testing for coronary artery disease on angiography.

Search methods MEDLINE (January 1966-November 2009) and EMBASE (1980-2009) databases were searched for articles on diagnostic accuracy of exercise stress testing.

Selection criteria We included prospective studies comparing exercise stress testing with a reference standard of coronary angiography in patients without known coronary artery disease. Results From 6055 records, we included 34 studies with 3352 participants. Overall, we found published studies regarding five different exercise testing modalities: treadmill ECG, treadmill echo, bicycle ECG, bicycle echo and myocardial perfusion imaging. The prevalence of $\mathrm{CAD}$ ranged from $12 \%$ to $83 \%$. Positive and negative likelihood ratios of stress testing increased in low prevalence settings. Treadmill echo testing $(\mathrm{LR}+=7.94)$ performed better than treadmill ECG testing $(\mathrm{LR}+3.57)$ for ruling in $\mathrm{CAD}$ and ruling out $\mathrm{CAD}$ (echo LR$=0.19$ vs ECG $L R-=0.38)$. Bicycle echo testing $(\mathrm{LR}+=11.34)$ performed better than treadmill echo testing (LR $+=7.94$ ), which outperformed both treadmill ECG and bicycle ECG. A positive exercise test is more helpful in younger patients $(L R+=4.74)$ than in older patients ( $\mathrm{LR}+=2.8$ ).

Conclusions The diagnostic accuracy of exercise testing varies, depending upon the age, sex and clinical characteristics of the patient, prevalence of $\mathrm{CAD}$, and modality of test used. Exercise testing, whether by echocardiography or ECG, is more useful at excluding $\mathrm{CAD}$ than confirming it. Clinicians have concentrated on individualising the treatment of $\mathrm{CAD}$, but there is great scope for individualising the diagnosis of CAD using exercise testing.

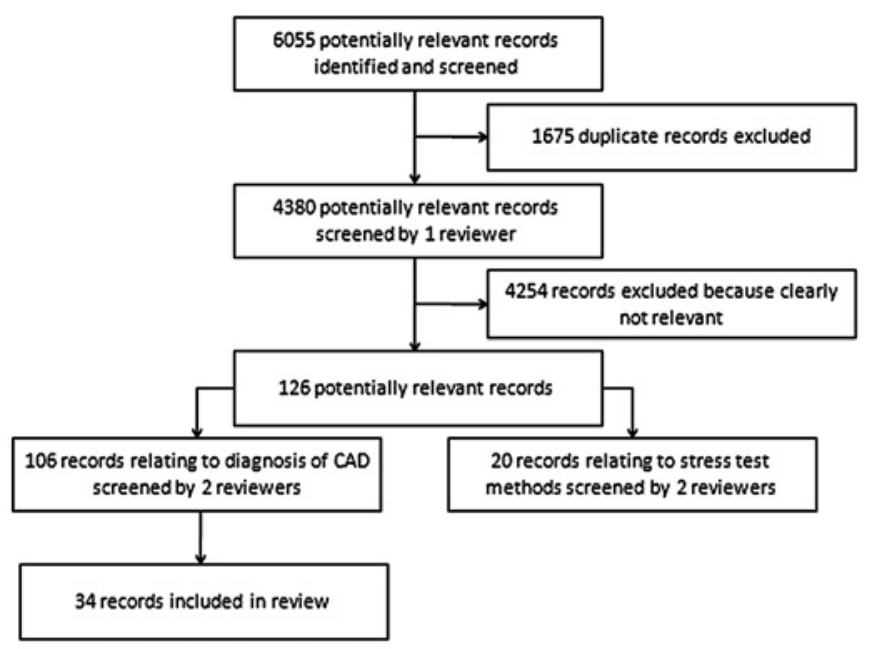

Abstract 64 Figure 1

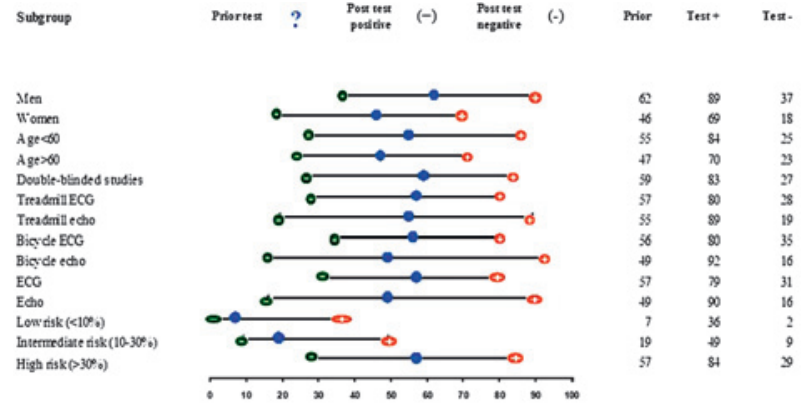

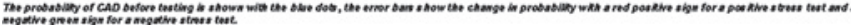

Abstract 64 Figure 2 Probability of coronary artery disease.

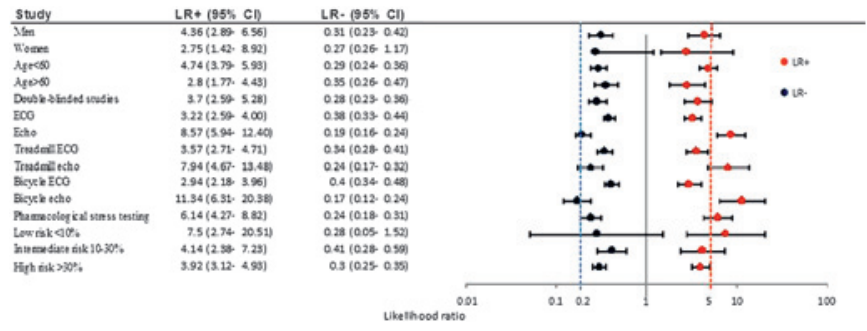

Abstract 64 Figure 3

\section{OUTCOMES AFTER CARDIAC SURGERY: ARE WOMEN OF SOUTH ASIAN ORIGIN AT INCREASED RISK?}

doi:10.1136/heartjnl-2011-300198.65

${ }^{1} \mathrm{D}$ A George, ${ }^{1} \mathrm{D}$ Morrice, ${ }^{2} \mathrm{~A}$ M Nevill, ${ }^{1} \mathrm{M}$ Bhabra. ${ }^{1}$ New Cross Hospital, Wolverhampton, UK; ${ }^{2}$ University of Wolverhampton, Wolverhampton, UK

Objectives The population served by our centre has a relatively high proportion of people originating from the Indian subcontinent ("South Asians") compared to the national average (14.3\% vs $4.6 \%$ ). We observed that the mortality rate in South Asian women undergoing cardiac surgery in our unit appeared to be relatively high. We investigated this observation further to determine whether ethnic origin was an independent risk factor for postoperative death in females.

Methods Data for all patients undergoing cardiac surgery were collected prospectively in a registry. Retrospective analysis was carried using SPSS on data for 4901 patients operated on in the 6year period April 2004 to March 2010. Categorical data associated with mortality were analysed using $\chi^{2}$ tests. Risk factors for inhospital mortality were subjected to univariate analysis, and those found to be significant were tested for independence using multivariate logistic regression.

Results During the study period, 1160 female patients underwent surgery with a mortality rate of $4.7 \%$. Mortality in 113 South Asians was $8.9 \%$ vs $4.3 \%$ in non-Asians ( $p=0.03$ ). Of 20 risk factors tested with univariate analysis, 16 were significantly associated with mortality. Logistic regression showed the following to be independent predictors of postoperative mortality: urgency of operation (OR 32.0; $\mathrm{p}<0.001$ ), older age (OR 24.2; $\mathrm{p}<0.001$ ), preoperative renal dysfunction (OR 15.8; $p<0.001$ ), diabetes (OR 7.8; $p=0.005$ ), South Asian ethnicity (OR 7.3; $\mathrm{p}=0.007)$, extra-cardiac arteriopathy (OR 4.8; $p=0.028$ ), and an operation other than isolated CABG (OR $5.8 ; \mathrm{p}=0.016)$.

Conclusions In our population, South Asian ethnicity appears to be an independent risk factor for mortality in females undergoing cardiac surgery. Studies in larger populations are warranted. 